\title{
Tunable hydrophobicity on fractal and micro-nanoscale hierarchical fracture surface of metallic glasses
}

\author{
Meng Gao, Dong Peng Wang, Yong Feng Huang, Sheng Meng, and Wei Hua Wang* \\ Institute of Physics, Chinese Academy of Sciences, Beijing 100 190, People's Republic \\ of China
}

A series of fractal, hierarchical fracture surfaces with a tunable hydrophobicity were designed and fabricated by simply three-point bending of metallic glasses (MGs) with different annealing treatments. We microscopically analyzed the structures of the fracture morphology on various fracture surfaces by scanning electron microscope and atomic force microscope, and find that the tunable hydrophobicity originates from the intrinsic micro-nanoscale hierarchical, two-level fractal fracture micro-structures in MGs. Our results may provide a strategy to fabricate the tunable wetting surface with combination of the excellent mechanical properties in MGs.

\footnotetext{
Keywords: Micro-nanoscale hierarchical fracture structures, spatial fractal distribution, tunable hydrophobicity, metallic glasses

* Corresponding author.

E-mail address:whw@iphy.ac.cn
} 


\section{Introduction}

Controlling the wettability of solid surfaces is an important issue attracting increasing interests from both fundamental research and practical applications, especially for superhydrophobic surfaces [1-3]. In nature, there are many biological materials which exhibit excellent surface superhydrophobic property, such as lotus leaves and beetle wings [4-5]. By mimicking the biological and hydrophobic micro-structures, artificial hydrophobic surfaces were fabricated by creating hierarchical structures on hydrophobic substrates and chemically modifying a hierarchical structured surface with low surface energy materials, such as polymers [6]. For the solids with high surface energy, such as metals and alloys, the superhydrophobic property is also obtained by laser treatment, solution-immersion and chemical reaction to fabricate a low surface energy layer on the surface [7-9]. However, these artificial superhydrophobic metallic surfaces are easily destroyed, leading to the decline and even disappearance of hydrophobicity due to their poor mechanical stability and wear resistance [10].

Metallic glasses (MGs) without long-range periodic structure endow them with excellent mechanical properties such as ultrahigh strength, high hardness, and high wear resistance, which have potential structural and functional applications [11-13]. Recently, a series of hydrophobic MGs were obtained by fabricating the microscopic hierarchical structure on surfaces by compression molding considering their excellent thermoplastic forming property [14-16]. However, the method has its limitations because some MGs such as Ce- and Sr-based MGs are readily destroyed and have poor mechanical stability; some MGs such as Pd-, Pt-based MGs are costly, which make them difficult to be applied in practice. Therefore, seeking an economic and applicable method to realize the combination of the excellent mechanical properties and the hydrophobic property of the MGs is challenging for practical applications.

Typical fracture morphology on fracture surface of MGs can be precisely tuned by structural relaxation and minor-alloying [17-18]. Recently, it is surprisingly found that fracture surface morphology shows the common fractal characteristic in various MG 
systems, which indicates that the fracture surface has a hierarchical micro-structure [19]. From this respect, these fracture surfaces of MGs with the stable mechanical properties and excellent wear resistance may be used for superhydrophobic surfaces. In this work, we report a series of hydrophobic fracture surfaces of the typical Zr-based MGs fabricated by simple three-point bending method. By precisely tuning the annealing states, various fracture surfaces with different wetting properties from near-superhydrophobic to hydrophilic are obtained. The readily obtained MG fractal fracture surfaces with stable and tunable hydrophobic property have potential applications in controlled heat transfer, self-cleaning surfaces.

\section{Experiment}

\subsection{Materials fabrication and characterization details}

Typical Zr-based MG $\left(\mathrm{Zr}_{52.5} \mathrm{Ti}_{5} \mathrm{Cu}_{17.9} \mathrm{Ni}_{14.6} \mathrm{Al}_{10}\right.$, Vit 105) as the model material for its good glass formation ability and great application potential was chosen in this work. The $5 \mathrm{~mm}$ MG rods were prepared from a master alloy with normal composition of $\mathrm{Zr}_{52.5} \mathrm{Ti}_{5} \mathrm{Cu}_{17.9} \mathrm{Ni}_{14.6} \mathrm{Al}_{10}$ by water-cooled copper mold casting. Its glass nature was checked by X-ray diffraction and differential scanning calorimetry. The MG rods were cut into a length of $30 \mathrm{~mm}$ for three-point bending tests. To precisely and systematically tune the fracture surface morphology, some three-point bending specimens encapsulated in a quartz crucible with vacuum of $10^{-4} \mathrm{~Pa}$ were annealed at $554 \mathrm{~K}\left(0.82 T_{g}\right)$ with different annealing times. The annealing times were chosen as 0 $\min$ (the as-cast state), $20 \mathrm{~min}, 40 \mathrm{~min}, 60 \mathrm{~min}, 120 \mathrm{~min}, 240 \mathrm{~min}, 360 \mathrm{~min}, 480 \mathrm{~min}$ and $14400 \mathrm{~min}$. Before three-point bending, the notches $(250 \mu \mathrm{m}$ in width and $1.5 \mathrm{~mm}$ in depth) were introduced into the middle of these rod-like samples by a diamond saw for confirming the flatness of fracture surfaces. The three-point bending tests were carried out in an Instron 3384 machine (Norwood, MA) with a cross-head moving speed of $0.5 \mathrm{~mm} \mathrm{~min}^{-1}$ at room temperature. The generated fracture surfaces were observed by scanning electron microscopy (SEM) conducted in a Philips XL30 instrument and atomic force microscope (MFP-3D-SA AFM) with nano-scale roughness. 


\subsection{Analysis methods}

To understand the relationship between the wetting property of the fracture surfaces and the fracture micro-structures, the fracture micro-structures were statistically analyzed by the fractal analysis method in the micro- and nano-scales. For micro-sale fracture structures, the most common method in the fractal analysis, the box-counting method is used to estimate the fractal dimension of the dimple nework-like structure on the fracture surface of metallic glasses [19]. For grids of square boxes with edge length $\Delta x$, the number of $N(\Delta x)$ of boxes covering at least one pixel of the binary black-and-white image corresponding to the original gray-scale SEM micrograph of the dimple structure has the power-law relation of $\mathrm{N}(\Delta \mathrm{x}) \sim \Delta \mathrm{x}^{-D_{\mathrm{f}}}$, where $D_{B}$ is a typical non-integer number (the physically meaningful values lie between one and two in two dimensions (2D), between two and three in 3D) called "box-counting" dimension for a fractal object. In log-log plot of $N(\Delta x)-\Delta x$, the slope is the fractal dimension $D_{f}$. For nano-scale structures, the AFM original height data was analyzed and got the spatial distribution function of micro-structure height.

\subsection{Wetting analysis}

The water contact angles (CAs) of the fabricated surfaces were measured by a contact angle system (OCA20, DataPhysics) and a charge coupled device camera system at room temperature. The probe liquid in measurement is ultra-pure water and the volume is about $0.5 \mu \mathrm{L}$. The measurement of contact angle for every surface was repeated three times to ensure the credibility.

\section{Results and discussion}

\subsection{SEM and AFM characterization of various metallic glass fracture surfaces}

Typical SEM and AFM images of the MG fracture surfaces at different annealing states including the as-cast state were shown in Fig. 1. Fig. 1 (a) exhibits macroscopic morphology of various fracture surfaces corresponding to different annealing states and almost keep flat by intentionally controlling the curvature radius of pre-notches. Especially, for the fracture surface with the annealing time of $14400 \mathrm{~min}$, the fracture 
surface is mirror-like, which is the typical fracture surface for brittle MGs [20]. The fracture morphology in different regions of a fracture surface corresponding to an annealing state are almost same, which shows the fractal nature of fracture surfaces [21]. The magnified fracture morphology of the regions marked by the black dashed circles in Fig. 1 (a) corresponding to different MG samples annealing at $554 \mathrm{~K}$ in different annealing times were displayed in Fig. 1 (b). It is obvious that fracture morphology (from as-cast state to the annealing state with the annealing time of 240 min) displays a complex dimple structure in Fig. 1 (a), which has been verified as the fractal network-like structures and the universal phenomena in various MGs [19, 22]. This unique fracture surface morphology reminds us of the universal leaf vein structures in nature, which have excellent hydrophobic and photosynthesis properties. The difference of the various dimple-like fracture surfaces lies in the average length scale of the whole fracture morphology and it decreases with the increase of annealing time, as is shown in Fig. 1 (b). For the annealing state with the annealing time of $14400 \mathrm{~min}$, a periodic stripe-like fracture structure appears in the mirror-like fracture surface compared with the dimple structures. Thus, one can see that various fracture morphology in MGs can be tuned by precisely designing the annealing temperature and the annealing time. Actually, as a special micronano-lab, various glassy nanostructures such as nanoridges, nanocones, nanowires and nanospheres could be formed on fracture surface by controlling the crack tip plastic zone [23], which provides the potential MG fracture surface applications.

Fig.1 (c) shows similar nanoscale hill-like protrusions appearing in the internal of dimples of different fracture surfaces with different spatial scales and spatial distributions. For as-cast state [the first picture of Fig. 1 (c)], the protrusions on fracture surface are more heterogeneous than those of the annealed samples [see Fig. 1 (c)] and the average height of protrusions decreases with the increase of annealing time. Compared to the nanoscale protrusion in dimple structures, the nanoscale periodic stripe exhibits a shallow sinusoidal-like shape [the last picture of Fig.1 (c)]. Based on the SEM and AFM results, one can see that the fracture surface of MGs 
displays the micro-nanoscale hierarchical structural characteristic, and the fracture surface morphology can be precisely tuned by proper annealing treatment. It is known that the wettability of a solid surface is usually governed by its surface energy and surface geometrical structure. For MGs, high surface energy makes it difficult to realize the hydrophobic property on their metallic surfaces. Recently, it is demonstrated that superhydrophobic metallic surfaces can be fabricated by the construction of multi-scale roughness [24-28]. The hierarchical structures, such as the overhang structures, can prevent water from penetrating the texture arising from the capillary force. Thus, those hierarchical MG fracture surfaces with micro and nano structures may be a candidate for hydrophobic surfaces.

\subsection{Wetting analysis of various metallic glass surfaces}

Figure 2 (a) shows a series of wetting analysis by a water drop $(<0.5 \mu \mathrm{L})$ on the various MG surfaces, including the polished surface, as-cast sample fracture surface and the annealed sample fracture surfaces with different annealing times corresponding to the states from state 0 to state 5 in Fig. 2 (b). The polished MG surface displays the normal wetting condition of metallic surfaces [the first picture of Fig. 2 (a)] and the contact angle is just $87.5^{\circ}$ in Fig.2 (b). Surprisingly, the fracture surface of as-cast MG shows the obvious hydrophobic property compared to that of the polished surface [the second picture of Fig. 2 (a)] and the contact angle reaches about $134.4^{\circ}$, which is very close to the contact angle limit of superhydrophobic surfaces. This result confirms the possibility of MG fracture surface as hydrophobic surface. What is more, with the increase of annealing time, the contact angle of fracture surface gradually decreases from $134.4^{\circ}$ (state 1) to $86.4^{\circ}$ (state 4 ) being close to the contact angle of the polished surface, which ranges from the near-superhydrophobic to normal-hydrophobic [see from Fig. 2 (a) and (b)]. And the contact angle of fracture surface for deeply annealed state 5 even decreases to $50.2^{\circ}$, which is below that of polished MG surface, as shown in the last picture of Fig. 2 (a). The wettability of MG fracture surfaces endures a transition from near-superhydrophobic to normal-hydrophobic and then transforms into 
normal-hydrophilic just by controlling the annealing. The corresponding contact angle ranges from $134.4^{\circ}$ to $50.2^{\circ}$ with a wide span of near $80^{\circ}$. Compared to the complex process and the highly demand fabricating the hierarchical micro-structures by chemical coating and thermoplastic forming process [15, 29], the fracture surfaces with different wetting properties and huge contact angle span tuned by annealing are simple and have a large potential in practice.

\subsection{Mechanical stability and durability test of various metallic glass fracture surfaces}

Mechanical stability and durability of various MG fracture surfaces with hydrophobic property was tested by scratch test. The methodology of scratch test is illustrated in Fig. 2 (c), which has been verified as an effective method [15]. MG fracture surface is moved back and forth with a speed of $5 \mathrm{~cm} / \mathrm{s}$ on the sandpaper (1000 mesh) under a constant pressure of $15 \mathrm{KPa}$. As is shown in Fig. 2 (d), after being abraded repeatedly for 200 cycles, the contact angles of various MG fracture surface show different and slight decreases compared to those before being abraded and the range of the contact angle changes is between $3{ }^{\circ}$ and $5^{\circ}$, which indicates that all MG fracture surface have a good mechanical stability and wear resistance. The inserted graph of Fig. 2 (d) gives the wetting comparison between before and after the scratch test of as-cast MG fracture surface. Considering the unique amorphous structure and metallic bonding, MGs have much higher hardness and strength and then own a higher abrasion resistance and mechanical stability, which is the main obstacle for most of natural and artificial superhydrophobic surfaces such as lotus leaf and organic modified surface with low surface energy [6-9]. The fracture micro-structures inherit these excellent mechanical properties during fracture and display outstanding advantages over other artificial superhydrophobic surfaces. What is more, considering the annealing-induced embrittlement effect on the strength and plasticity of MGs [30], the effect of the scratch corrosion on the hydrophobic property of deeply annealed MG fracture surface should be more obvious than the as-cast and moderate annealed ones, which is demonstrated by the red arrow line in Fig. 2 (d). However, it is noted that the scratch corrosion does not largely weaken the 
hydrophobic property of annealed MG fracture surfaces since the annealed MG fracture surfaces still keep the good mechanical properties [30].

\subsection{Statistical analysis of fracture structure for various metallic glass surfaces in} micro- and nano-scale

We systematically studied the fracture micro-structure evolution for various fracture surfaces in different scales, and the results are shown in Fig. 3 (a) and 3 (b). For annealed MGs, the micron-scale fracture surface morphology has a transition from the vein and dimple pattern to the periodic stripe pattern due to the evolution of intrinsic dynamic defects of flow units in MGs [17]. Therefore, the wetting property transition of annealed samples should be related to the micro-scale fracture morphology evolution. We then studied the evolution progress of typical micro-scale fracture morphology in Fig. 3 (a). In the three-point bending stress field, the typical fracture morphology of MGs is the dimple structures. In the double-log graph of dimple size $D$ and spatial distribution function $p(D)$ [see Fig. 3 (a)], there is a evident transition from the power-law distribution to Gaussian distribution when the annealing time increases to $120 \mathrm{~min}$ at $0.8 T_{\mathrm{g}}$. The transition corresponds well to the contact angle suddenly drops from $123^{\circ}$ to $109^{\circ}$ when the annealing time increases to $120 \mathrm{~min}$ in Fig. 2 (b). This indicates that the spatial distribution of the micro-scale dimples indeed affects the wettability of the fracture surface, and the fractal distribution of dimples is more favorable to form the hydrophobic property than the Gaussian distribution. Based on the AFM results, there exists the nanoscale protrusions in the internal part of dimples [see Fig. 1 (c)], which is analogous to nanoscale protrusions in the lotus leaves. In Fig. 3 (b), we examined the spatial evolution of nanoscale protrusion height in dimples and found a similar transition from the power-law distribution to Gaussian distribution when the annealing time increases to $120 \mathrm{~min}$ at $0.8 T_{g}$. Considering the formation of dimple structure [19], the consistency of spatial distribution transition of micro-nanoscale fracture structures could be understood and it is the nanoscale protrusions that congregate into the 
microscale dimples. Fig. 3 (c) shows the correlation between the contact angles $\theta$ and the fractal dimensions $D_{f}$ of microscale dimples and nanoscale protrusions for different fracture surfaces. The contact angle is nearly proportional to the fractal dimension and the double fractal fracture structures consisting of nanoscale protrusions on the microscale fractal and network-like dimple structures prefer to be hydrophobic. Similar phenomena can also be found in a large number of natural leaves [28], the typical fractal structure in nature. The superhydrophobicity of natural leaves is ascribed to the hierarchical structures on the surface consisting of randomly distributed micropapillae covered by branch-like nanostructures [28]. Our results present the quantitative correlation between the wetting property and the fractal dimension of microstructure, which is helpful for artificial superhydrophobic surfaces.

We note that with the continue increase of the annealing time, the spatial distribution becomes more close to the Gaussian distribution and the fracture micro-structure becomes more homogeneous [see Fig. 1 (b) and 1 (c)]. When the annealing time reaches a critical value, the periodic stripe pattern appears [see Fig. 1 (b) and 1 (c)]. Meanwhile, the contact angle decreases to $50.2^{\circ}$ and is lower $27.3^{\circ}$ than that of the polished surface, which is suggestive for realizing the further hydrophilicity of metallic surfaces. This stripe structure has an orientation order and breaks the interface capillary force balance between the air and the fracture surface, which leads to the collapse of the hydrophobicity. This result enlightens us that the different wettability of metallic surfaces may be tuned by fabricating the oriented micro-structures. Our results show that the fracture surfaces with double-fractal hierarchical structures indeed own the near-superhydrophobic property and the various wettability could be got by controlling the hierarchical fracture structures induced by proper annealing treatment. To clarify the physical mechanism of wettability of fracture surfaces of MGs, we proposed a sketch of various MG surfaces with different fracture micro-structures in Fig. 4. Fig. 4 (a) shows the normal wetting property for polished surfaces (contact angle $\theta<90^{\circ}$ ) and Fig. 4 (b) gives the near-superhydrophobic double-fractal fracture surfaces (contact angle $\theta<120^{\circ}$ ). With 
the increase of annealing times, the double-fractal fracture surface becomes homogeneous and the wettability transforms into the normal hydrophobicity (contact angle $90^{\circ}<\theta<120^{\circ}$ ) [see Fig. (c)]. When the dimples transforms into the periodic stripes, the wettability appears a sudden transition from the hydrophobicity to the hydrophilicity and the hydrophilicity is even stronger than the intrinsic MG surface (contact angle $\theta<50^{\circ}$ ). The sketch might give a new scheme for designing the superhydrophobic and superhydrophilic metallic surfaces and provide some enlightenment for understanding the physical mechanism of wettability of metallic surfaces.

\section{Conclusions}

In this study, a series of MG fracture surfaces with various wetting properties were specially designed and fabricated by simple annealing treatment and three-point bending method and then statistically analyzed. The following conclusions were obtained:

1. The metallic glass fracture surfaces corresponding to different annealing states display the huge wetting transition from near-superhydrophobic to near-superhydrophilic.

2. The spatial distribution evolution of micro-nanoscale structures governs the huge wetting transition for various metallic glass fracture surfaces. The as-cast metallic glass fracture surface with double-fractal hierarchical dimple structures exhibits the surprising near-superhydrophobic property and the deeply annealing metallic glass fracture surface with periodical stripe structures exhibits near-superhydrophilic property.

3. For metallic glass fracture surfaces with typical dimple pattern, the contact angle in wetting analysis shows a good positive correlation with the fractal dimension of fracture surfaces. This result suggests that the complication and fractal composition of surface are prone to display the hydrophobic character. 
4. The designing scheme fabricating the hydrophobic surfaces largely extends the practical applications of bulk MGs in a wide range of different fields. This method could be widely adopted for the fabrication of other BMG surfaces with special wettability.

\section{Acknowledgments}

The work is supported by the NSF of China (Grant No. 51271195 and 5141101072) and the MOST 973 Program (No. 2015CB856800). The discussions and experimental help of D. Q. Zhao, and valuable discussions with Prof. H. Y. Bai and M. X. Pan are appreciated.

\section{References}

[1] R. Wang, K. Hashimoto, A. Fujishima, M. Chikuni, E. Kojima, A. Kitamura, M. Shimohigoshi, T. Watanabe, Light-Induced Amphi-philic Surfaces, Nature 388 (1997) 431-432.

[2] R. Blossey, Self-cleaning surfaces-virtual realities, Nat. Mater. 2 (2003) 301-306.

[3] J. Aizenberg, P. Fratzl, Biological and biomimetic materials, Adv. Mater. 21 (2009) 387-388.

[4] B. Bhushan, Y.C. Jung, Natural and biomimetic artificial surfaces for superhydrophobicity, self-cleaning, low adhesion, and drag reduction, Prog. Mater. Sci. 56 (2011) 1-108.

[5] K. Liu, X. Yao, L. Jiang, Recent developments in bio-inspired special wettability, Chem. Soc. Rev. 39 (2010) 3240-3255.

[6] Z.G. Guo, W.M. Liu, B.L. Su, Superhydrophobic surfaces: From natural to biomimetic to functional, J. Colloid. Interf. Sci. 353 (2011) 335-355.

[7] Z.G. Guo, F. Zhou, J.C. Hao, W.M. Liu, Stable biomimetic super-hydrophobic engineering materials, J. Am. Chem. Soc. 127 (2005) 15670-15671. 
[8] S.T. Wang, L. Feng, L. Jiang, One-step solution-immersion process for the fabrication of stable bionic superhydrophobic surfaces, Adv. Mater. 18 (2006) 767-770.

[9] G. Godeau, C.R. Szczepanski, T. Darmanin, F. Guittard, Nanoparticle covered surface: An efficient way to enhance superhydrophobic properties, Mater. Des., 92 (2016) 911-918.

[10] K.S. Liu, L. Jiang, Metallic surfaces with special wettability, Nanoscale, 3 (2011) $825-838$

[11] H.B. Yu, Y.S. Luo, K. Samwer, Ultrastable metallic glasses, Adv. Mater. 25 (2013) 5904-5908

[12] R.C. Sekol, G. Kumar, M. Carmo, F. Gittleson, N. Hardesty-Dyck, S. Mukherjee, J. Schroers, A.D. Taylor, Bulk metallic glass micro fuel cell, Small 9 (2013) 2081-2085.

[13] M.W. Chen, A brief overview of bulk metallic glasses, NPG Asia Mater. 3 (2011) $82-90$.

[14] M. Hasan, J. Schroers, G. Kumar, Functionalization of metallic glasses through hierarchical patterning, Nano Lett. 15 (2015) 963-968.

[15] J. Ma, X.Y. Zhang, D.P. Wang, D.Q. Zhao, D.W. Ding, K. Liu, W. H. Wang, Superhydrophobic metallic glass surface with superior mechanical stability and corrosion resistance, Appl. Phys. Lett. 104 (2014) 173701.

[16] W.H. Wang, Bulk metallic glasses with functional physical properties, Adv. Mater. 21 (2009) 4524-4544.

[17] G. Li, M.Q. Jiang, F. Jiang, L. He, J. Sun, The ductile to brittle transition behavior in a Zr-based bulk metallic glass, Mater. Sci. Eng., A 625 (2015) $393-402$

[18] N. Nollmann, I. Binkowski, V. Schmidt, H. Rosner, G. Wilde, Impact of minor-alloying on the plasticity of Pd-based bulk metallic glasses, Scr. Mater. 111 (2016) 119-122.

[19] M. Gao, B.A. Sun, C.C. Yuan, J. Ma, W.H. Wang, Hidden order in the frcature surface morphology of metallic glasses, Acta Mater. 60 (2012) 6952-6960. 
[20] G. Wang, Y.T. Wang, Y.H. Liu, M.X. Pan, D.Q. Zhao, W. H. Wang, Evolution of nanoscale morphology on fracture surface of brittle metallic glass, Appl. Phys. Lett. 89 (2006) 121909.

[21] B.B. Mandelbrot, D.E. Passoja, A.J. Paullay, Fractal character of fracture surfaces of metals, Nature 308 (1984) 721-722.

[22] E. Bouchaud, D. Boivin, J.L. Pouchou, D. Bonamy, B. Poon, G. Ravichandran, Fracture through cavitation in a metallic glass, Europhys. Lett, 83 (2008) 66006.

[23] X.X. Xia, W.H. Wang, A. L. Greer, Plastic zone at crack tip: A nanolab for formation and study of metallic glassy nanostructures, J. Mater. Res. 24 (2009) 2986-2992.

[24] N.Li, T. Xia, L. Heng, L. Liu, Bio-inspired multifunctional metallic glass, Appl. Phys. Lett. 102 (2013) 251603.

[25] T. Xia, N. Li, Y. Wu, L. Liu, Patterned superhydrophobic surface based on Pd-based metallic glass, Appl. Phys. Lett. 101 (2012) 081601.

[26] F.Z. Dai, D.P. Wen, Y.K. Zhang, J.Z. Lu, X.D. Ren, J.Z. Zhou, Micro-dimple array fabricated on surface of Ti6Al4V with a masked laser ablation method in air and water, Mater. Des. 84 (2015) 178-184.

[28] L. Cao, H.H. Hu, D. Gao, Design and fabrication of micro-textures for inducing a superhydrophobic behavior on hydrophilic materials, Langmuir 23 (2007) 4310-4314.

[29] K.S. Liu, Z. Li, W.H. Wang, L. Jiang, Facile creation of bio-inspired superhydrophobic Ce-based metallic glass surfaces, Appl. Phys. Lett. 99 (2011) 261905.

[30] W.D. Li, Y.F. Gao, H.B. Bei, On the correlation between microscopic structural heterogeneity and embrittlement behavior in metallic glasses, Sci. Rep. 5 (2015) 14786 


\section{Figures captions}

Fig.1. (a) Overview of various fracture surfaces of Vit 105 after three-point bending fracture in different annealing times at $554 \mathrm{~K}$ (from left to right): As-cast (0 min); 20 min; $60 \mathrm{~min} ; 240 \mathrm{~min} ; 14400 \mathrm{~min}$. (b) High-magnification detailed microgragh corresponding to the regions marked by the black-dashed circles in Fig. 1 (a). (c) AFM images of fracture micro-structures corresponding to the regions marked by the red-dashed circles in Fig. 1 (b). Unit of data bars is nm.

Fig.2. (a) Contact angle images of various MG surfaces including the polished surface (0) and fracture surfaces corresponding to different annealing states marked with 1,2, 3, 4 and 5 with different annealing times: As-cast ( $0 \mathrm{~min}$ ); $20 \mathrm{~min}$; $60 \mathrm{~min}$; $240 \mathrm{~min}$; 14400 min, respectively. (b) Correlation between contact angles, wettability and annealing times for different MG surfaces. (c) Illustration of the scratch test for MG fracture surface. (d) Contact angle changes of various MG fracture surfaces after scratch tests. The red arrow line shows the contact angle change evolution with the increasing of the annealing time and the inserted graph gives the wetting comparison between before and after the scratch test of as-cast MG fracture surface.

Fig.3. (a) Spatial distribution evolution of typical fracture micro-structure-dimple size with different annealing times. (b) Probability distribution evolution of nanoscale protrusion height in one dimple structure with different annealing times. (c) Correlation between the fractal dimension of dimple size and nanoscale protrusion height distribution and the corresponding contact angle.

Fig.4. Sketch of the various wetting properties of different MG surfaces: (a) MG polished surface; (b) As-cast MG fracture surface (double-fractal dimple structure); (c) Annealed MG fracture surface I in moderate annealing (homogeneous dimple structure); (d) annealed MG fracture surface II in deeply annealing (periodic stripe structure). 

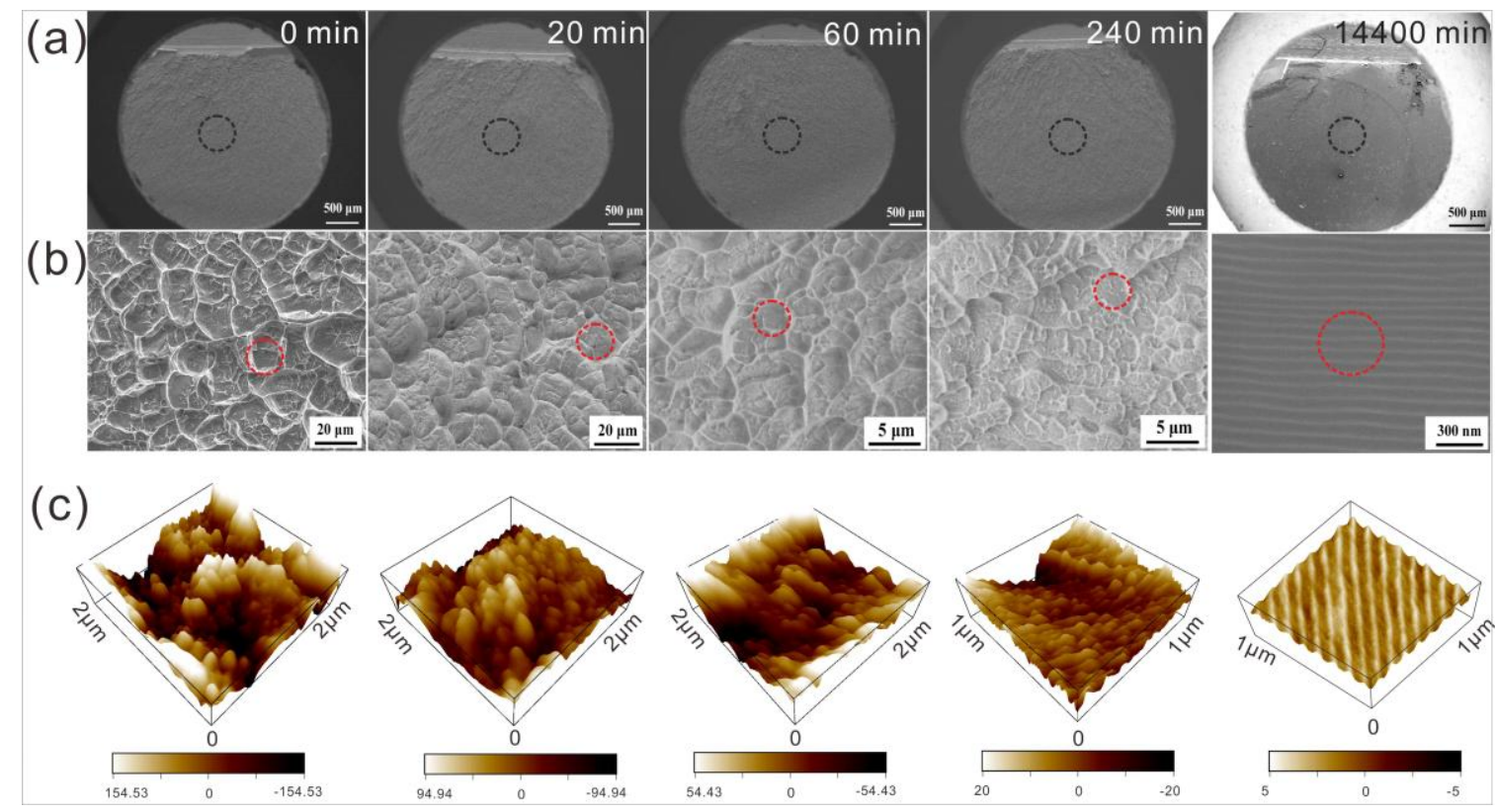

Fig. 1, Gao et al. 

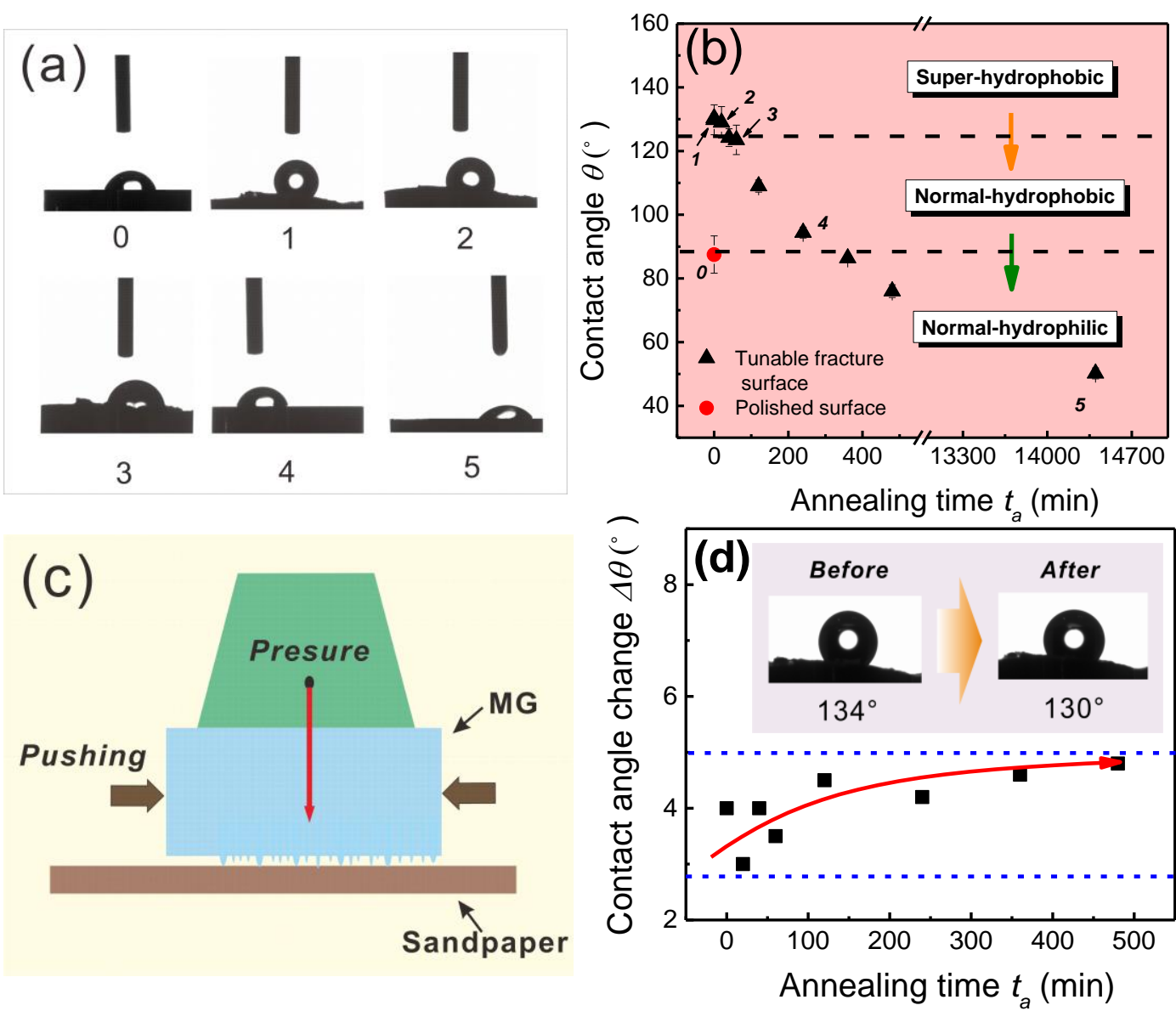

Fig. 2, Gao et al. 

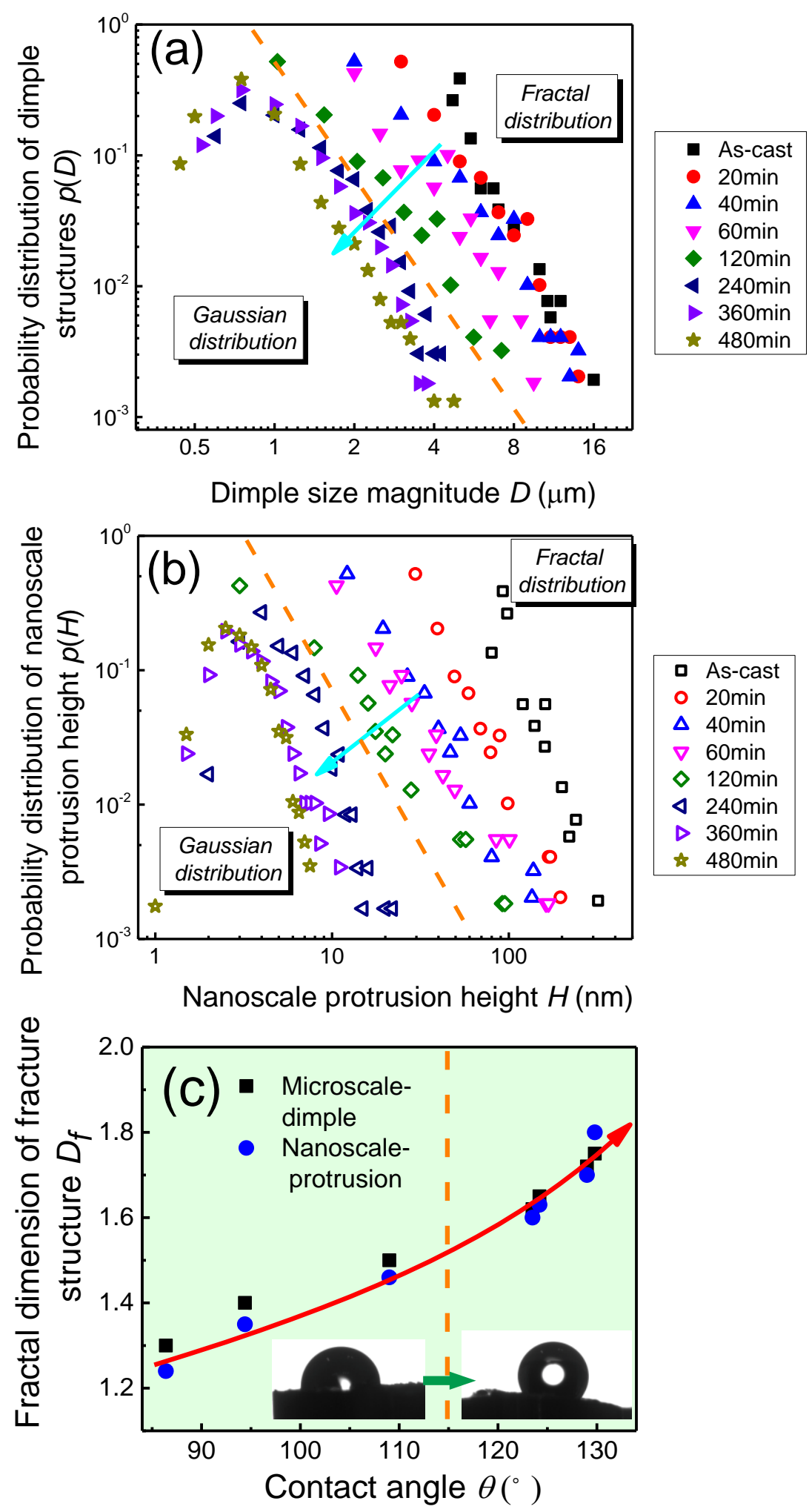

Fig. 3, Gao et al. 
(a) MG polished surface

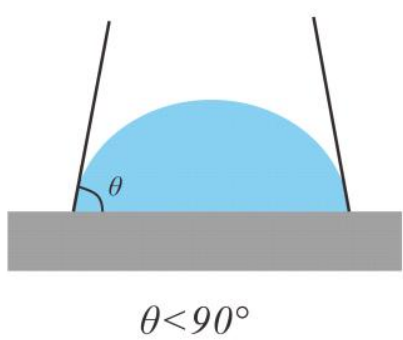

(C) Annealed MG fracture

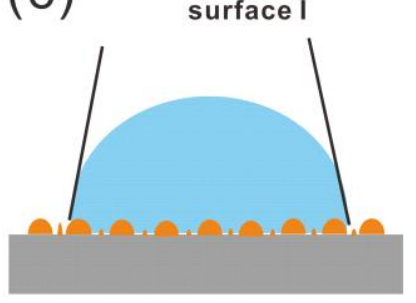

$\theta<90^{\circ}$ (b) $\begin{gathered}\text { As-cast MG } \\ \text { fracture surface }\end{gathered}$

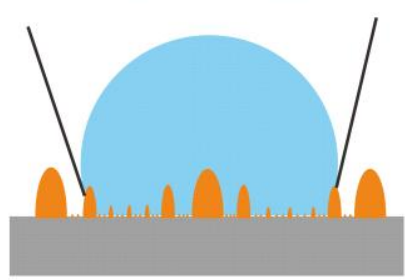

$\theta>120^{\circ}$

(d) $\begin{gathered}\text { Annealed MG fracture } \\ \text { surface II }\end{gathered}$

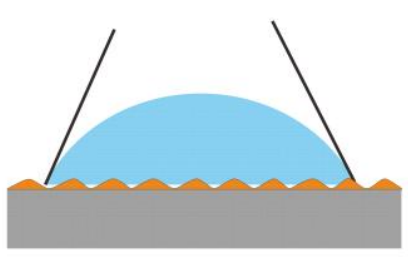

$\theta<50^{\circ}$

Fig. 4, Gao et al. 


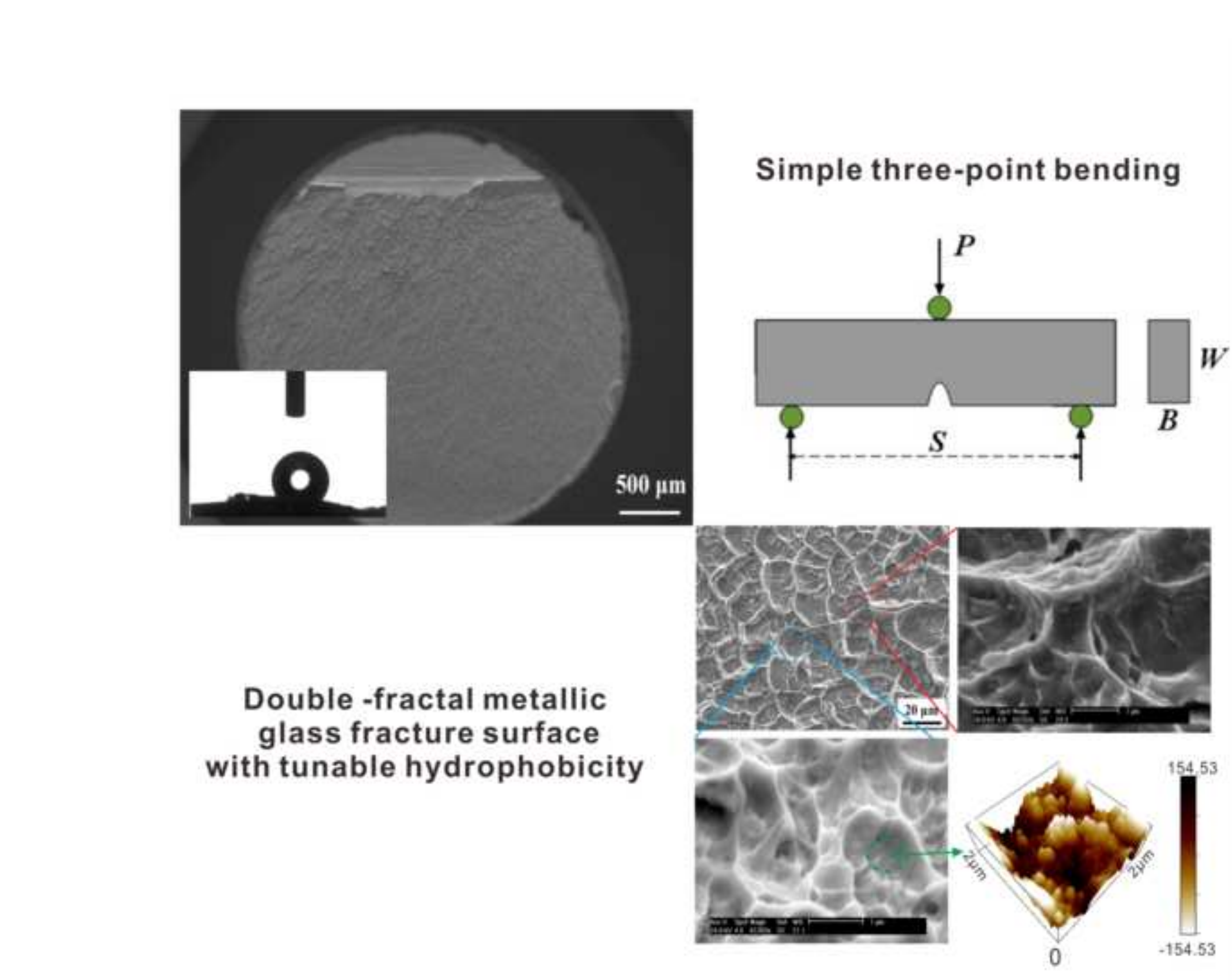

Simple three-point bending

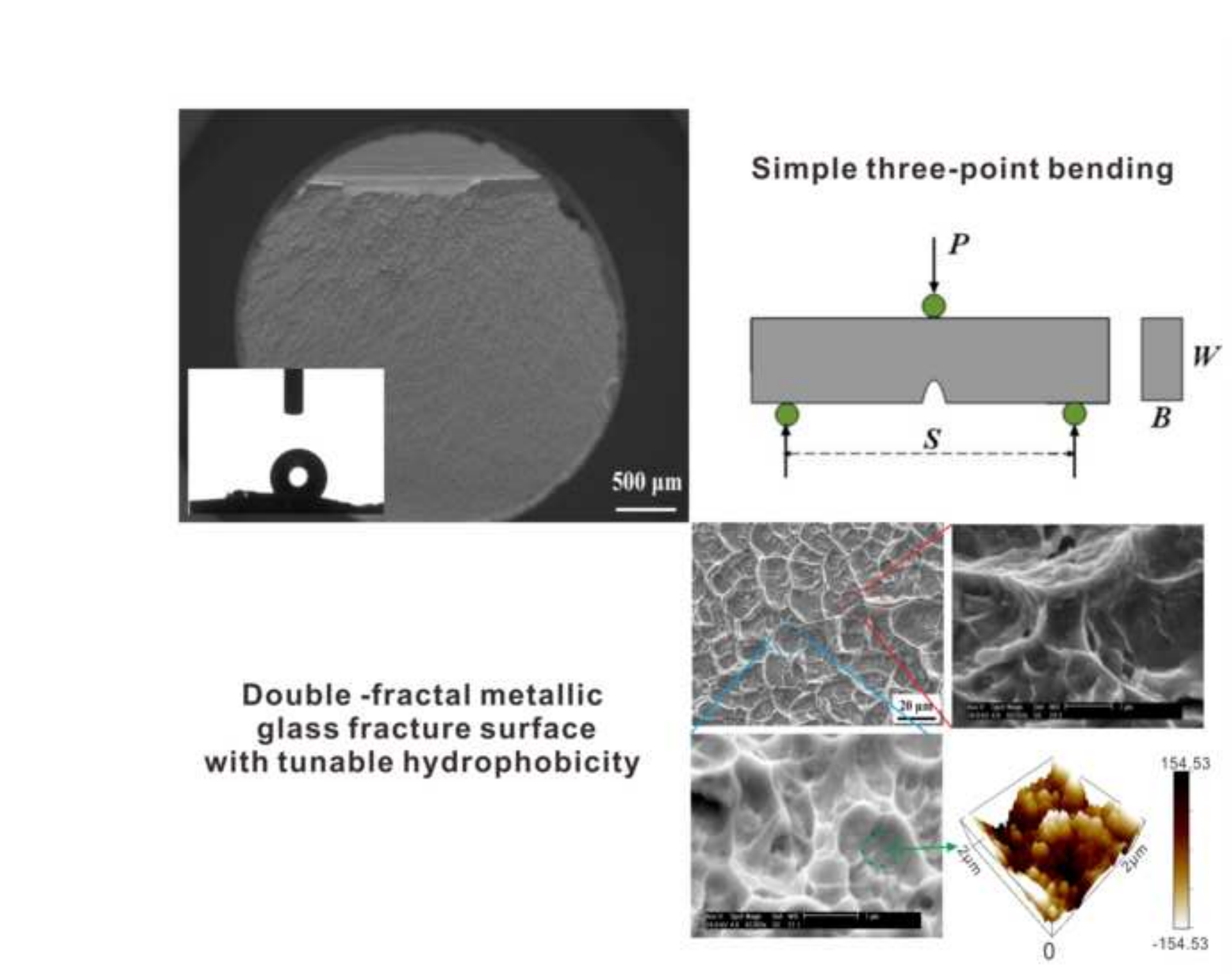

Double -fractal metallic

glass fracture surface with tunable hydrophobicity

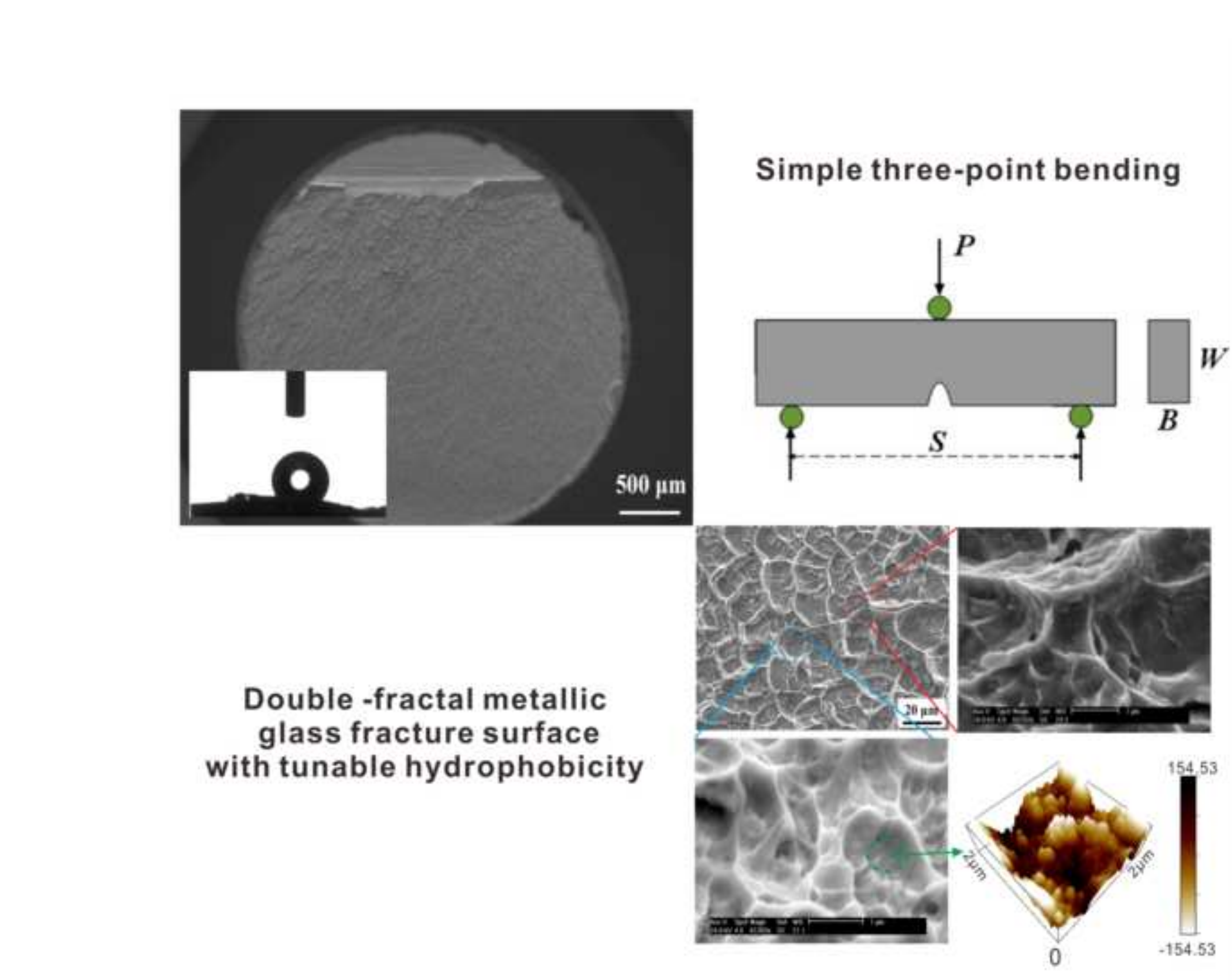

